\title{
Tuberculosis in Children Living amongst Adults with Tuberculosis at the Tuberculosis and Leprosy Referral Hospital, Eku, Nigeria
}

\author{
Atana Uket Ewa ${ }^{1}$, Dominic Fabian Essiet ${ }^{2}$, Samuel John Umejei Monu ${ }^{3}$ \\ ${ }^{1}$ Department of Paediatrics, University of Calabar/Teaching Hospital, Calabar, Nigeria \\ ${ }^{2}$ Formerly, Department of Haematology, Baptist Hospital, Eku, Nigeria \\ ${ }^{3}$ Formerly, Delta State Tuberculosis and Leprosy Control Program/Tuberculosis and Leprosy Referral Hospital, \\ Eku, Nigeria \\ Email: ${ }^{*}$ atanaewa@yahoo.com
}

Received 18 June 2015; accepted 25 August 2015; published 28 August 2015

Copyright (C) 2015 by authors and Scientific Research Publishing Inc.

This work is licensed under the Creative Commons Attribution International License (CC BY).

http://creativecommons.org/licenses/by/4.0/

(c) (i) 0 pen Access

\section{Abstract}

Background: Tuberculosis (tb) causes significant morbidity and mortality worldwide. In Nigeria, the exact prevalence is not known but a progressive increase of the disease has been observed, with "epidemic states" reported in certain clusters. Even with the newer diagnostic tests for tb, diagnostic difficulties still occur. As a result, several diagnostic criteria have been adopted to aid the diagnosis of tuberculosis in these children. The main objective of this study was to identify Tb in children who were either living with adults who have Tb or in the environment of adults with Tb. Methodology: This was a prospective cross-sectional study conducted in Eku and Oria communities in Ethiope East Local Government Area of Delta State. Eku housed the Tuberculosis and Leprosy Referral Hospital where adults with tb were admitted in the first two months of their treatment. 128 children under 19 years of age were studied over a 3-month period. After obtaining relevant information from every child, a full physical examination and certain laboratory investigations were done. Results: There were 68 males and 60 females. The prevalence of Tb was $68 \%$ in those children living among adults with tb within the hospital and $50.6 \%$ in those living in the environment or community $(p=0.05)$. The prevalence of $\mathrm{Tb}$ in Bacille Calmette Guerin (BCG) immunized children was $66.2 \%$ as against $46.7 \%$ in those not vaccinated. Twenty-six of the infected children $(57.8 \%)$ had only pulmonary disease while $45.7 \%$ had affectation of 2 or more organs. The positive predictive values of elevated erythrocyte sedimented rate (ESR), elevated white blood cells (WBC) count and positive gastric aspirates (GA) in diagnosing tb were $60.3 \%, 70.5 \%$ and $0 \%$ respectively, while that of abnormal chest X-ray (CXR) and positive Mantoux reaction were $93.6 \%$ and $93.7 \%$ respectively. Conclusion: The alarming prevalence of tb in these children was not surprising considering their contacts with the tb patients and was representative of simi-

\footnotetext{
${ }^{*}$ Corresponding author.

How to cite this paper: Ewa, A.U., Essiet, D.F. and Monu, S.J.U. (2015) Tuberculosis in Children Living amongst Adults with Tuberculosis at the Tuberculosis and Leprosy Referral Hospital, Eku, Nigeria. Journal of Tuberculosis Research, 3, 80-89. http://dx.doi.org/10.4236/jtr.2015.33013
} 
lar high incidence areas as a whole. Active case finding efforts should be intensified for childhood contacts of adults with $\mathrm{Tb}$ for early identification of children with tuberculosis.

\author{
Keywords
}

Tuberculosis, Case Finding, Positive Predictive Values, Contacts

\title{
1. Introduction
}

Tuberculosis (tb) is a disease commonly caused by Mycobacterium tuberculosis (M. tuberculosis) [1] [2]. It is an old disease, with known aetiology, diagnosis and treatment using known drugs that have not changed much over the years [3] [4]. Historically, even though tb existed as far back as 100BC and 700AD, it was not a major health problem until after $1600 \mathrm{AD}$, when the industrial revolution began [5] [6]. Man then began to live in densely populated cities, creating ideal conditions for air-borne spread of M. tuberculosis. Then, for the first time in world history, tuberculosis became epidemic, but did not reach the interior of Africa until about 1910 [5]. It has since remained a serious, though preventable disease, in the developing countries of Africa [7] [8]. Over the past decades, there has been an upsurge of tuberculosis in several parts of the world [1] [2]. One of the epidemiological indicators used to measure the magnitude and trends of tuberculosis is the prevalence of infection and the tuberculin positivity of a cross-section of the population predicts this [9]. Data from the prevalence of infection indicate that in low income countries, the prevalence of infection in children entering school is commonly around 10\% - 25\% [10] [11]. In Nigeria, the exact prevalence of tuberculosis is not known [12] [13] but a progressive increase of the disease has been observed, with "epidemic states" of the disease reported in certain areas [12].

The diagnosis of $\mathrm{Tb}$ in children is not precise [14]. It is based on clinical findings and a high index of suspicion by the clinician [14]. Two situations cause the clinician to suspect Tb. The first occurs when a child has contact with an adult with $\mathrm{Tb}$. The tuberculin test is reactive, chest radiograph (CXR) and/or physical examination leads to the discovery of early disease. The second situation is when $\mathrm{Tb}$ is part of the differential diagnosis for an ill child [15]. Owing to the difficulties in confirming childhood tuberculosis even with availabilty of newer diagnostic tests [16], various criteria have been proposed to help to improve the control of tuberculosis in the communities [17]-[20].

The Nigerian Tuberculosis and Leprosy Control Programme (NTBLCP) had in the past recommended a combination of criteria for diagnosing tuberculosis in children. These included clinical history, family contact history, X-ray examination and tuberculin testing; among others [21]. This score chart may be useful when the diagnosis of tuberculosis is difficult in children [22]. It is basically the same as the Papua New Guinea scoring scheme, with a score of 7 or more indicating a high likelihood of tuberculosis. The main advantage over the World Health Organisation (WHO), Lusaka, Addis Ababa and the Pakistani criteria is that it does not need the radiological or bacteriological services to meet the diagnosis, which makes it good for the developing countries. But it has the disadvantage of being made for use in children with advanced disease as well as a high risk of over diagnosing tb in developing countries, where malnutrition with or without underlying HIV infection prevails. Unfortunately, these international and local criteria continue to lack uniformity and acceptance, creating the need to intensify efforts to developing more reliable methods for diagnosing paediatric tuberculosis infection and/or disease [23].

This highlighted the need to explore the available diagnostic combinations of history, clinical and radiological findings, elevated erythrocyte sedimentation rate (ESR), positive PPDMantoux test and elevated white blood cell (WBC) count. This suggested criteria that incorporated the WBC modified the Ethiopian criteria [18].

The aim of this study was to identify and describe tuberculosis in the studied children and also to determine

1) The prevalence of tuberculosis in these children;

2) The prevalence of tuberculosis in the children with BCG immunization;

3) Pattern of tuberculosis infection in both BCG immunized and non-immunized children;

4) Common site of involvement of tuberculosis in these children;

5) Predictive value of the baseline tests used in the diagnosis of tuberculosis in these children. 


\section{Methodology}

The study was conducted over a three-month period (January to March 2001) in Eku and Oria communities of Delta state of Nigeria, using the Tuberculosis and Leprosy Referral Hospital (TBLRH). The selected communities were chosen because Eku housed the TBLRH as its major district hospital and is about $3 \mathrm{~km}$ from Oria. People from both communities of Eku and Oria attended the hospital for other ailments and some of the children came to the hospital compound to play. In the TBLRH project at the time of study, tuberculous patients were admitted during the first two months of treatment and some came to the wards with their young infants and children. The children in the study had the highest risk of acquisition of tuberculosis through close contact with adult sufferers of tuberculosis disease. Contact investigation for children in these families had not yet been programmed for on the TBLCP project and as such was not implemented at that time. The sample size for this study was determined [24] [25] and it was projected that a total number of 128 children would be studied. Ethical clearance was obtained from the University of Benin Teaching Hospital (UBTH) Ethical committee. Permission was also obtained from the Delta State Ministry of Health as well as authorities of the TBLRH, Eku. Consent was obtained from parents of the studied children in both communities.

All children of adult cases of $\mathrm{Tb}$ and those from within and around the referral hospital were enumerated and listed in an orderly sequence. Using a table of random numbers, one child per adult case of $\mathrm{Tb}$ was randomly selected. Thereafter, in order to achieve the rest of the sample size and to ensure a reasonable representation and spread, a blocked randomization technique was utilized to select the children from Eku and Oria communities. The households were enumerated and listed in an orderly manner and the number of children per household were equally enumerated and listed in an orderly sequence. The sampling interval of one of every five household was utilized to identify the study households and one child per study household was selected using a table of random numbers.

Relevant information obtained on every child included name, date of birth, sex, address, religion, ethnic group, immunization status giving date of BCG vaccination, date of recruitment, duration of breast feeding, parent's occupation and educational level, family income, number of siblings, recent illnesses, household contact with an adult with tuberculosis, as well as present complaints, after which a full physical examination was done on each child.

Mantoux test was done using an intradermal injection of $0.1 \mathrm{ml}$ of 10IU of purified protein derivative (PPD), which was read after 72 hours [15] [26]. Those children with abnormal physical findings and/or positive tuberculin reactors further had full blood count and erythrocyte sedimentation rate (ESR). The normal ESR value used for children was less than $10 \mathrm{~mm} / \mathrm{hr}$ [27].

Chest radiographs and gastric aspirates were done on selected children who were sick or in whom other investigation results were abnormal. These children were made to fast prior to each visit for early morning gastric aspirations, which were done on three consecutive days as day cases [28]. Cases were stratified by social class according to their parent's characteristics using a social score index as described by Olusanya et al. [29].

Data were analysed using Epi info software. Chi Square and 2-tailed Fisher's exact test were used for comparisons. The p-value was significant if $<0.05$. If the expected numbers had a value less than 5 , Fisher's exact test was used. Also, if the Cornfield confidence interval for Odds Ratio at 95\% did not include 1.0, the association was statistically significant.

\section{Results}

A total of 128 children under 19 years were studied. Male: Female ratio was 1:0.9. Their ages ranged from 0.41 to 18.95 years with a mean of 9.3 years and a median of 9.9 years. The children within the hospital living among adults with tuberculosis were 47 (36.7\% of the total) while those in the community living in the environment of adults with tuberculosis were 81 (63.3\%). Tuberculosis affected mainly children from lower socioeconomic classes. There were 75 (58.6\%) cases with abnormal physical signs. Symptoms included cough which was found in 49 (38.3\%) as well as fever and weight loss which were each present in 8 (6.3\%). Forty-seven (36.7\%) children had lymphadenitis and these involved mainly the cervical regions. Two (1.6\%) children each had rhonchi and crepitations in the chest while only one had abdominal distension. Hepatomegaly was found in 47 children while 24 had splenomegaly. The combination of hepatosplenomegaly was found in 11 children. The commonest site of involvement of tb was the lungs with associated cervical lymphadenopathy. 


\subsection{Diagnosis of Tuberculosis}

Tuberculosis was diagnosed following the discovery of abnormal physical signs and/or positive Mantoux test as well as using a minimum of three criteria. These included elevated ESR $>10 \mathrm{~mm} / \mathrm{hr}$, abnormal CXR, elevated WBC $\geq 10,000 / \mathrm{mm}^{3}$ and positive gastric aspirates for acid fast bacilli (AFB). Their sensitivities, specificities and positive predictive values (PPV) are shown (Figure 1). Abnormal CXR and positive Mantoux reaction maintained very good sensitivities and adequate specificities and ppv as shown (Figure 1).

\subsection{The Prevalence of Tuberculosis}

The prevalence of $\mathrm{Tb}$ in all the children $<19$ yrs was $57 \%$. The prevalence of $\mathrm{Tb}$ in children within the hospital, living among adults with $\mathrm{Tb}$ was found to be $68 \%$ while that of children in the environment (community) was $50.6 \%$. This difference was statistically significant at Chi Square $=3.70$ and p value $=0.0543$. CI for Odds Ratio at $95 \%$ was 2.08 (0.92 - 4.74). The prevalence of Tb in BCG immunized children was $66.2 \%$ as against $46.7 \%$ in those not vaccinated (Table 1). This difference was statistically significant with Chi Square $=3.89$ and $\mathrm{p}$ value $=0.049$.

\subsection{Combinations of Diagnostic Criteria}

Abnormal physical signs, when used alone in diagnosing Tb gave very poor sensitivity (46.7\%), specificity (32.2\%) and ppv (46.7\%). But these improved when used with other combinations. When abnormal clinical history, positive family contact history, abnormal X-ray examination and positive tuberculin test were combined for diagnosis, only 14 (19.2\%) of the 73 children with probable Tb were identified. A combination of any 3 parameters diagnosed 35 (48\%) of the children with probable $\mathrm{Tb}$. Nineteen $(26 \%)$ of the $\mathrm{Tb}$ cases were diagnosed using any 2 criteria. But when abnormal CXR and positive Mantoux were specifically chosen, 57 (78.1\%) were identified with Tb. Only 5 (6.8\%) were identified using any one criterion, but positive Mantoux alone identified

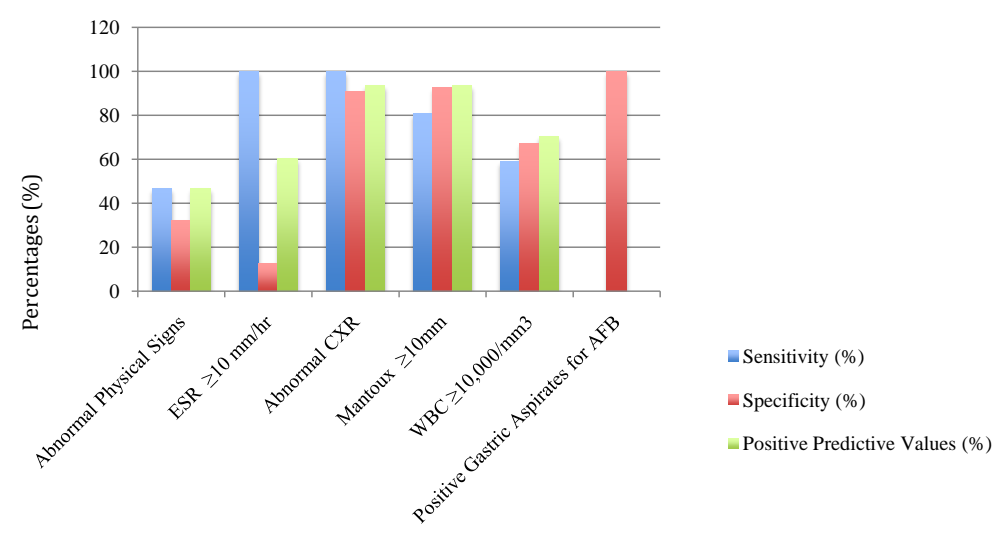

Figure 1. The sensitivity, specificity and positive predictive value for each criterion for diagnosing tuberculosis. Where: ESR-Erythrocyte Sedimentation Rate, WBC—White blood cell count, GA—Gastric Aspirate, CXR—Chest X-ray.

Table 1. Prevalence of $\mathrm{Tb}$ in the general population.

\begin{tabular}{ccc}
\hline Age in yrs & BCG vaccinees $(\mathrm{a} / \mathrm{x}$ in\%) & Non vaccinees $(\mathrm{b} / \mathrm{y}$ in \%) \\
\hline $0-<5$ & $11 / 19(57.9) 5 / 19(26.3)$ & $8 / 13(61.5)$ \\
$5-<10$ & $14 / 20(70)$ & $8 / 19(42.1)$ \\
$10-<15$ & $13 / 20(65)$ & $7 / 9(77.8) 7 / 9(77.8)$ \\
$15-<19$ & $45 / 68(66.2) 28 / 60(46.7)$ & \\
Total &
\end{tabular}

Where: $\mathrm{a}$ is $\mathrm{Tb}$ in the vaccinees, $\mathrm{b}$ is $\mathrm{Tb}$ in non vaccinees, $\mathrm{x}$ is number of vaccinees and $\mathrm{y}$ is number of unvaccinated children. 
54 (74\%) while abnormal CXR used alone identified 73 (100\%) with Tb. Abnormal physical signs were in turn combined with one, two and three laboratory tests. Good predictive values were found with combinations of abnormal physical findings and positive Mantoux alone, abnormal CXR alone, abnormal WBC alone, ESR and WBC, ESR and Mantoux, CXR and WBC as well as CXR, Mantoux and ESR (Table 2).

\subsection{Mantoux Results}

The overall Mantoux reactions ranged from 0 - $22 \mathrm{~mm}$ with a mean of $8 \mathrm{~mm}$ and a median of 9 mm. Thirty seven (54.4\%) of the children who received BCG vaccination had Mantoux reactions more than 10 mm while the remaining $45.6 \%$ had negative reactions. Thirty-one (75.6\%) of 41 children with household contact who had diagnosed Tb were Mantoux positive; 30 (73.2\%) of these fulfilled the study criteria for the diagnosis of tuberculosis. Out of the 47 children with cervical lymphadenopathy, 66\% were Mantoux positive and 35 (74.5\%) had probable tuberculosis. The prevalence of $\mathrm{Tb}$ in children with cervical adenopathy was higher than in their counterparts without adenopathy (74.5\% vs 46.9\%, $\chi^{2}=9.21, \mathrm{p}=0.0024$, Odds Ratio $=3.30$, CL = 1.41 - 7.85). Thirty nine (52\%) children with other abnormal physical signs had positive Mantoux reactions as against 36 (48\%) who had Mantoux reaction less than $10 \mathrm{~mm}\left(\chi^{2}=0.56, \mathrm{p}=0.45\right)$.

\subsection{Comparative Studies}

The BCG vaccination rate in children under 19 years was 53.1\%, with scar formation occurring in $75 \%$ of those who received BCG. The pattern of Tb infection in both BCG immunized and non-immunized children living in the hospital among adults with $\mathrm{Tb}(\mathrm{n}=32)$ was initially similar to that of the general population. Before 5 years, the prevalence of $\mathrm{Tb}$ was $25 \%$ in non-vaccinees and $77.8 \%$ in the vaccinated. Between 5 and 10 years, this rose to $80.0 \%$ in the non-vaccinees and to a greater extent (83.3\%) in those vaccinated. After 10 years but before 15 years, this prevalence started dropping to $70 \%$ in the vaccinees and $25 \%$ in the unvaccinated group, to reach a low level of $66.7 \%$ and $0 \%$ in the vaccinated and non-vaccinated groups respectively. When the Tb prevalence was compared in each of the different age groups between the vaccinees and non-vaccinees, the differences were not statistically significant (p values for Chi Square ranged from 0.07 and 0.8 ).

The presence of a positive Mantoux reaction was also compared in the BCG vaccinated and unvaccinated children across age groups (Table 3). For children under 5 years of age, the prevalence of positive reaction was much higher in the vaccinated than the unvaccinated (31.6\% vs $10.5 \%, \chi^{2}=2.53, \mathrm{p}=0.111$ ). Thereafter, both groups of children, had an almost similar sharp and sustained rise with a plateau in the vaccinated but dropped momentarily in the unvaccinated at $10-14$ years $\left(60 \%\right.$ vs $\left.47.4 \%, \chi^{2}=0.63, p=0.43\right)$. The two unvaccinated children under 5 years with positive Mantoux reaction both developed $\mathrm{Tb}$.

The Mantoux responses in the BCG vaccinated were further analyzed. Positive and negative responses were compared among the different age groups. About 21\% of under fives were Mantoux positive among whom 7

Table 2. Combinations of abnormal physical signs with various criteria.

\begin{tabular}{|c|c|c|c|}
\hline $\begin{array}{l}\text { Abnormal physical signs } \\
\text { with criteria }\end{array}$ & $\begin{array}{c}\text { Sensitivity } \\
\text { (\%) }\end{array}$ & $\begin{array}{l}\text { Specificity } \\
\text { (\%) }\end{array}$ & $\begin{array}{c}\text { Positive } \\
\text { predictive value (\%) }\end{array}$ \\
\hline Mantoux $\geq 10 \mathrm{~mm}$ & 76.6 & 88.5 & 92.3 \\
\hline $\mathrm{ESR} \geq 10 \mathrm{~mm} / \mathrm{hr}$ & 100 & 32 & 74.2 \\
\hline Abnormal CXR & 100 & 65.7 & 77.8 \\
\hline $\mathrm{WBC} \geq 10,000$ & 60.4 & 72 & 80.6 \\
\hline Positive GA & 0 & 100 & 0 \\
\hline ESR and Mantoux & 88.3 & 60.3 & 83.3 \\
\hline ESR and WBC & 80.2 & 52 & 77.4 \\
\hline ESR and Positive GA & 50 & 66 & 37.1 \\
\hline ESR and CXR & 100 & 48.9 & 76 \\
\hline WBC and Positive GA & 30.2 & 86 & 40.3 \\
\hline CXR and WBC & 80.2 & 68.9 & 79.2 \\
\hline CXR and Positive GA & 50 & 82.9 & 38.9 \\
\hline CXR, Mantoux and ESR & 92.2 & 62.1 & 81.4 \\
\hline
\end{tabular}

Where: ESR—Erythrocyte Sedimentation Rate, WBC—White blood cell count, GA—Gastric Aspirate, CXR—Chest X-ray. 
Table 3. Prevalence of positive Mantoux reactions in both vaccinees and non-vaccinees.

\begin{tabular}{|c|c|c|}
\hline Age in yrs & BCG vaccinated $(\mathrm{a} / \mathrm{x}$ in\%) & Non vaccinated (b/y in \%) \\
\hline $0-<5$ & 6/19 (31.6) & 2/19 (10.5) \\
\hline $5-<10$ & $12 / 20(60.0)$ & 8/13 (61.5) \\
\hline $10-<15$ & $12 / 20(60.0)$ & 9/19 (47.4) \\
\hline $15-<19$ & 7/9 (77.8) & 7/9 (77.8) \\
\hline Total & \multicolumn{2}{|c|}{$37 / 68(54.4)$ 26/60 (43.3) } \\
\hline
\end{tabular}

Where: $\mathrm{a}$ is positive Mantoux in the vaccinees, $\mathrm{b}$ is positive Mantoux in non vaccinees, $\mathrm{x}$ is number of vaccinees and $\mathrm{y}$ is number of unvaccinated children.

5.0\% had received BCG. From 5 years to less than 10 yrs, 60.6\% were Mantoux positive among whom $60 \%$ had received BCG vaccination. After 9 yrs, 53.9\% were tuberculin positive among whom 57.1\% were vaccinated. After 14 years, $77.8 \%$ were tuberculin positive and among them $50 \%$ were vaccinated. The association between the presence of positive Mantoux among the vaccinees and unvaccinated in all age groups below 15 years had $p$ values for Chi Square between 0.1 and 0.9 .

The corollary findings were that 13 out of the 30 tuberculin negative under fives had received BCG; 8 out of 13 tuberculin negative children 5 to 10 years old were vaccinated. About forty four percent of tuberculin negative 10 - 14 year old were vaccinated while $50 \%$ of tuberculin negative 15 to 18 years olds had received BCG. There was no response in $8.2 \%$ of the children who had probable Tb.

\section{Discussion}

The prevalence of tuberculosis in the study children was 57\%. This was even higher (68\%) among the children within the hospital who were living with adults with tuberculosis. It is therefore not surprising that this prevalence is much higher than those from previous studies, which gave a range of $10 \%$ - 25\% [1] [10] [11] [25].

While comparing the prevalence of positive Mantoux reactions in the vaccinees and unvaccinated of the same age group, this study showed the prevalence to be much higher in the vaccinees below 5 years of age than their unvaccinated counterparts (31.6\% vs $10.5 \%$ ). Thereafter the prevalence was similar in both groups but the rise was more sustained in the vaccinees than their counterparts. An increase in tuberculin sensitivity with increasing age has also been observed in Nigeria [30] and elsewhere [31] [32]. This demonstrated the potentiating effect of mass BCG vaccination of under-fives on immunity that was previously primed by environmental mycobacteria in those children [31]. and is possibly explained by an increased exposure to the Tb bacilli with increasing age [31]-[34]. This finding agrees with that of other studies where a subsequent and steady rise in skin reactivity in the vaccinated was compared to the non-vaccinated children [31] [32]. Although the difference in the rise of tuberculin reactivity between the two groups was not statistically significant, the observed trends might suggest a waning effect of the BCG in those older than 5 years of age. Other studies have shown the waning effect of BCG with age [30]-[33] [35].

The rise in tuberculin reactivity with age could not be attributed to the direct effect of BCG, which should have manifested sooner after vaccination, or to infection with $M$. Tuberculosis, which should have affected the non-vaccinated and the vaccinated groups equally. The explanation may be as previously thought that BCG induced immunity as well as skin reactivity to tuberculin are enhanced by contact with environmental mycobacteria [31] [32]. The significance of this is that vaccinees show an increased ability to respond immunologically to subsequent encounters with environmental mycobacteria [32]. The results of this study strongly support the expectations of these postulates. Age and community of residence are strong predictors of a positive reaction. Other studies comparing tuberculin sensitivities in the vaccinees and non-vaccinees have concluded that past BCG vaccination grossly enhanced the prevalence of tuberculin sensitivities in a geographical location [31].

For those children living in the hospital among tuberculous adults, the prevalence was initially similar to the general population. It was lower in the vaccinees below 10 years of age compared to the unvaccinated, but became higher after 10 years in the vaccinees than the unvaccinated. This probably supports the hypothesis that varying levels of immunity are mediated by natural exposure to environmental mycobacteria [36]. In this study, $75 \%$ of the 68 children who had received BCG developed scars. Evidence from the literature agrees that not all 
vaccinees usually develop scars and indicates that the rate of scar development varies [31]. Among Indian children living in Canada, 78\% of vaccinees developed visible scars [31]. In South Africa, scar formation could be detected in 38\% of children confirmed to have received BCG [37]. In Kenya, BCG produced a scar in over $80 \%$ of the vaccinees, while elsewhere in Nigeria, BCG scar was detected in $44 \%$ of children vaccinated [38]. The presence of a scar has been accepted as evidence of efficacious vaccination and considerable protection [37]. Unfortunately, tuberculin hypersensitivity or scar formation does not necessarily reflect protection against disease. In this study, 45 of the 73 children diagnosed with Tb actually received BCG with scar formed in 40 of them. The main aim of neonatal BCG is to prevent disseminated forms of Tb such as tuberculous meningitis (TbM) and miliary $\mathrm{Tb}$ [37]. In this study, none of the vaccinated children who had scars and had Tb suffered from TbM or the disseminated forms of $\mathrm{Tb}$.

Six (6) out of 73 (8.2\%) cases of tuberculosis did not show any response to the Mantoux test. This agrees with studies, which showed that about $10 \%$ of immunocompetent children with culture proven Tb did not react initially to the tuberculin test. Most became reactive after several months of treatment [15]. The tuberculin test may be used to select persons who require BCG vaccination as those with no Mantoux response who did not have Tb were given BCG. It is also a useful tool in the diagnosis and control of Tb [33] [39].

A history of household contact of adult Tb was obtained in 41 (32\%) of the study children and $75.6 \%$ of them were tuberculin positive. This is higher than earlier studies, which had observed an average of $30 \%-50 \%$ of household contacts who were Mantoux positive [2]. Whereas children who are close contacts of adult tb cases are known to develop tb [4] [11] [40]-[42], thirty (73.2\%) of the studied household contacts had tuberculosis while the remaining $26.8 \%$ had not. Therefore, it is not all children who share a common source of contact that ever develop $\mathrm{Tb}$. These differences in susceptibility to Tb among animals and humans suggest the existence of a genetically determined ability to resist $\mathrm{Tb}[43]-[45]$.

In this study, 93.6\% of the 78 cases with abnormal CXR were diagnosed with $\mathrm{Tb}$. There was no case of $\mathrm{Tb}$ with a normal CXR. However, studies have shown that a normal CXR does not exclude miliary tuberculosis and that miliary changes may develop within several days [14]. On the other hand, significant abnormalities on chest radiograph have been present in more than $50 \%$ of children with tuberculosis who have few or no symptoms [46]. The chest radiographs in this study had a sensitivity of $100 \%$, a specificity of $90.7 \%$ and a positive predictive value of $93.5 \%$. It is recommended for routine use in diagnosing childhood tuberculosis either singly or in combination. A combination of diagnostic criteria based on history of contact, reactive tuberculin, CXR and/or abnormal physical findings have been documented to lead to the discovery of early disease [15] [21]. This and other combinations as shown in this study provide greater strength for diagnosis than using single criteria. Also this contradicts findings from other studies which showed that the chest radiograph is often non-specific and only in about half of the cases will it be highly suspicious or diagnostic of pulmonary tuberculosis [14].

The commonest site of involvement of tuberculosis in the study children was the lungs, with drainage to the cervical nodes, with or without hepatosplenomegaly. Pulmonary tuberculosis is generally agreed to be the commonest type of tuberculosis in children [22]. Hepatosplenomegaly was a common finding in this study and its presence represented possible disseminated tuberculosis.

Forty eight percent (48\%) of the study children had cervical lymphadenitis. All cases of lymphadenitis involved the cervical group with $74.5 \%$ of them being found in children with probable tuberculosis. Other sites included the hilar and perihilar regions. Only one child had axillary lymph nodes in addition, and he was diagnosed with probable tuberculosis. Tuberculous lymphadenitis was found across the entire age range with over $60 \%$ involving the cervical nodes. This is not unexpected since the primary organs infected are the lungs, with drainage through the hilar nodes to the mediastinal, supraclavicular and other cervical lymphnodes. The axillary group, which are also close to the lungs were involved next in 21\% [47] It is possible that only one case of axillary lymphadenitis was found in this study because the Tb cases were detected early. It was interesting to note the absence of generalized lymphadenopathy in these children. This agrees with the work of Aderele [48] who found a paucity of generalized lymphadenitis in some Nigerian children with Tb. It is recommended that children with generalized lymphadenitis in this and similar environment are more likely to have diseases other than tuberculosis and should be fully investigated for them [48].

Gastric aspirates had no yield for AFB in the studied children. Gastric aspirate had a poor yield for AFB in childhood Tb in this and other studies [15]. Infants are most often unable to produce sputum and so gastric aspirates need to be collected [14]. These are seldom collected outside of referral hospitals and usually yield positive results in less than $30 \%$ of cases [14]. The newer diagnostic tests are specific but have limited sensitivity for di- 
agnosing tb from these samples, thereby creating an urgent need for improved diagnostic tests for tb in children [49]. However, acid-fast smears and culture remain the primary means of establishing the diagnosis of $\mathrm{Tb}$ in children, with culture being the gold standard for laboratory confirmation of tb [50].

The main strength of this study is early detection of tuberculosis in otherwise well children playing around the communities. A major limitation was the difficulty encountered in confirming tuberculosis with AFB and culture of gastric aspirates. It is generally known that GA gives a low yield of M. tuberculosis [15]. This was a community-based study on children at risk of acquiring tb, most of who were not yet "sick" with tb. It is possible that the yield may have been higher if it was a hospital-based study on "sick" children. Also, gastric aspirates were done on the children as day cases after a fast [28]. Perhaps the yield might have improved if they were admitted overnight but this was not feasible for this community-based study. Also, there is paucity of facilities to confirm tb with culture of gastric aspirates in this and several other developing countries.

\section{Conclusion}

This study has documented the true position of tuberculosis in children in these communities and in similar high incidence areas in Nigeria and other endemic countries. There should therefore be routine screening of children living in high prevalence areas. It has also shown that child contacts of adult tb cases can acquire the disease and hence national $\mathrm{Tb}$ control programmes should intensify efforts at active case finding of child contacts of adult tb cases. BCG vaccination of children who are Mantoux negative with no evidence of tuberculosis should be practiced so as to maintain the protection against severe forms of tb at all times. Similar studies should be repeated in other high prevalence areas using 5 iu of tuberculin and perhaps refine gastric aspirate collection protocols according to the current national guidelines to improve yield of AFB and also to document a more current epidemiological data. Again, considering the fact that not all children who came in contact with the tb bacilli developed the disease, further studies also need to be done to predict which contacts are likely to be protected and which ones are likely to develop the disease. Chemoprophylaxis could be prescribed to reduce illness and spread of infection. Finally, the effect of re-vaccination with BCG needs to be studied. It is possible that this might increase its long-term protection since its protective effect has been found to wane with time. Also, it is possible to improve on the BCG vaccine currently in use if we could understand and modify the adjuvant property rather than the antigenic content. A therapeutic vaccine that would prevent tb-infected individuals from becoming sick with the disease is the highest priority for vaccine research.

\section{References}

[1] Sudre, P., Dam, D. and Kochi, A. (1992) Tuberculosis: A Global Overview of the Situation Today. Bulletin of the WHO, 70, 149-159.

[2] Starke, J.R., Jacobs, R.F. and Jereb, J. (1992) Resurgence of Tuberculosis in Children. Journal of Pediatrics, 120, 839-855. http://dx.doi.org/10.1016/S0022-3476(05)81949-3

[3] Leung, A.N. (1999) State of the Art Reviews. Pulmonary Tuberculosis: The Essentials. Radiology, 210, 307-322. http://dx.doi.org/10.1148/radiology.210.2.r99ja34307

[4] Donald, P.R. (2013) History of Medicine: Edith Lincoln, an American Pioneer of Childhood Tuberculosis. The Pediatric Infectious Disease Journal, 32, 241-245.

[5] Stead, W.N., Eisenach, K.D., Cave, M.D., Beggs, M.L., Templeton, G.L., Theon, C.O. and Bates, J.H. (1995) When Did Mycobacteria tuberculosis Infection First Occur in the New World? American Journal of Respiratory and Critical Care Medicine, 151, 1267-1268.

[6] Pugliese, G. (1992) Screening for Tuberculosis: An Update. American Journal of Infection Control, 20, 37-42. http://dx.doi.org/10.1016/S0196-6553(05)80125-7

[7] Zumla, A., Raviglione, M., Hafner, R. and Fordham von Reyn, C. (2013) Current Concepts: Tuberculosis. New England Journal of Medicine, 368, 745-755. http://dx.doi.org/10.1056/NEJMra1200894

[8] Beza, M.G., Wubie, M.T., Teferi, M.D., Getahun, Y.S., Bogale, S.M. and Selam B. Tefera, S.B. (2013) A Five Years Tuberculosis Treatment Outcome at Kolla Diba Health Center, Dembia District, Northwest Ethiopia: A Retrospective Cross-Sectional Analysis. Journal of Infectious Diseases and Therapeutics, 1, 101-106.

[9] Dommisse, J. (1975) Undiagnosed Primary Tuberculosis as a Possible Major Cause of High Infant and Child Mortality Rate. J Trop Paediatr Envir Ch Health, 6, 21. http://dx.doi.org/10.1093/tropej/21.6.307

[10] Rider, H.L. (1997) Epidemiology of Tuberculosis in Children. Tuberculosis in Childhood. Annales Nestle. NESTEC 
Ltd., 55, 1-9.

[11] Payghan, B.S., Kadan, S.S. and Kotresh, M. (2013) The Prevalence of Pulmonary Tuberculosis among Severely Acute Malnourished Children-A Cross Sectional Study. International Journal of Scientific and Research Publications, 3, $1-5$.

[12] Williams, G.A., Benebo, N.S., Adeleye, M.O., Alabi, G.A. and Krishna, S.A.R. (1991) National Tuberculosis and Leprosy Control Programme: Worker's Manual.

[13] Federal Ministry of Health-Department of Primary Health Care and Disease Control (1999) National Tuberculosis and Leprosy Control Programme: Report of a Review of the National Tuberculosis Control in Nigeria.

[14] Schaaf, H.S., Gie, R.P., Beyers, N., Smuts, N. and Donald, P.R. (1993) Tuberculosis in Infants Less than 3 Months of Age. Archives of Disease in Childhood, 69, 371-374. http://dx.doi.org/10.1136/adc.69.3.371

[15] Stark, J.R. and Correa, A.G. (1995) Management of Mycobacterial Infection and Disease in Children. The Pediatric Infectious Disease Journal, 14, 455-470. http://dx.doi.org/10.1097/00006454-199506000-00001

[16] Eisenach, K.D., Cave, M.D., Bates, J.H. and Craford, J.T. (1990) Amplification of a Repetitive Sequence Specific for Mycobacterium Tuberculosis Using the Polymerase Chain Reaction. Journal of Infectious Diseases, 161, 977-981. http://dx.doi.org/10.1093/infdis/161.5.977

[17] Osborne, C.M. (1995) The Challenge of Diagnosing Tuberculosis in a Developing Country. Archives of Disease in Childhood, 72, 369-374. http://dx.doi.org/10.1136/adc.72.4.369

[18] Negusse, W. (1993) Bone and Joint Tuberculosis in Childhood in a Children's Hospital, Addis Ababa. Ethiopian Medical Journal, 31, 51-61.

[19] Qasi, S.A., Khan, S. and Khan, M.A. (1998) Epidemiology of Childhood Tuberculosis in a Hospital Setting. Journal of Pakistan Medical Association, 48, 90-93.

[20] Attawell, K., Smith, I., Janssen, P., Smith, S., Wikinson, D., Hudelson, P., Chondoka, S., Bwalya, P. and Sumatojo, E. (1996) Tuberculosis and Children: The Missing Diagnosis. Child Health Dial, 1-16.

[21] Federal Ministry of Health-Department of Primary Health Care and Disease Control (1998) Tuberculosis. National Tuberculosis and Leprosy Control Programme-Revised Worker's Manual, 1-4, 22-45, 164-185.

[22] Johnson, A.O.K. and Aderele, W.I. (1979) Abdominal Tuberculosis in Childhood. The Journal of Tropical Medicine and Hygiene, 82, 47-52.

[23] Anderson, S. and Gerser, A. (1960) The Distribution of Tuberculosis Infection among Households in Africa Communities. Bulletin of the World Health Organization, 22, 36-60.

[24] Lwanga, S.K. and Lameshow, S. (1991) Sample size Determination in Health Studies. WHO, Geneva.

[25] Khali, A.W. and Ahmed, T. (1993) The Continuing Challenge of Tuberculosis. Postgraduate Doctors, 5, 312-318.

[26] Chaulet, P. (1990) The Standardized Tuberculosis Test. In Childhood Tuberculosis,...Still with Us! WHO Document, 32-33.

[27] Al-Marri, M.R.H.A. and Kirkpatrick, M.B. (2000) Erythrocyte Sedimentation Rate in Childhood Tuberculosis: Is It Still Worthwhile? International Journal of Tuberculosis and Lung Disease, 4, 237-239.

[28] Lobeto, M.N., Loeffler, A.M., Furst, K., Cole, B. and Hopewell, P.C. (1998) Detection of Mycobacterium Tuberculosis in Gastric Aspirates Collected from Children: Hospitalization Is Not Necessary. Pediatrics, 102, e40. http://dx.doi.org/10.1542/peds.102.4.e40

[29] Olusanya, O., Okpere, E. and Ezimokhai, M. (1985) The Importance of Social Class in Voluntary Fertility Control in a Developing Country. West African Journal of Medicine, 4, 205-212.

[30] Ogunmekan, D.A. (1980) The Relationship between Age and Sensitization to PPD-S and Atypical Mycobacterial Antigens among School Children in Lagos, Nigeria. African Journal of Medicine and Medical Sciences, 9, 15-20.

[31] Young, T.K. and Mirdad, S. (1992) Determinants of Tuberculin Sensitivity in a Child Population Covered by Mass BCG Vaccination. Tubercle and Lung Disease, 73, 94-100. http://dx.doi.org/10.1016/0962-8479(92)90062-O

[32] Al-Kassim, F.A., Abdullah, A.K., Al-Orainey, I.O., Benar, A.B., Al-Hajjaj, M.S., Al-Majed, S. and Al-Wazzan, A. (1991) The Significance of Positive Mantoux Reaction in BCG Vaccinated Children. Tubercle, 72, 101-104. http://dx.doi.org/10.1016/0041-3879(91)90035-Q

[33] Mudido, P.M., Guwatudde, D., Nakakeeto, M.K., Bukenya, G.B., Nsamba, D., Johnson, J.L., Mugerwa, R.D., Ellner, J.J. and Whalen, C.C. (1999) The Effect of BCG Vaccination at Birth on Tuberculin Skin Test Reactivity in Ugandan Children. International Journal of Tuberculosis and Lung Disease, 3, 891-895.

[34] Stanford, J.L., Shield, M.J. and Rock, G.A.W. (1981) How Environmental Mycobacteria May Predetermine the Protective Efficacy of BCG. Tubercle, 62, 55-62. http://dx.doi.org/10.1016/0041-3879(81)90037-4

[35] Sterne, J.A.C., Rodrigues, L.C. and Guesdes, I.N. (1998) Does the Efficacy of BCG Decline with Time since Vaccina- 
tion? International Journal of Tuberculosis and Lung Disease, 2, 200-207.

[36] Fine, P.E.M. (1995) Variation in Protection by BCG: Implications of and for a Heterologous Immunity. Lancet, 346, 1339-1345. http://dx.doi.org/10.1016/S0140-6736(95)92348-9

[37] Donald, P.R., Van Zyl, L.E. and de Villiers, J. (1995) BCG Vaccination Status of Children with Tuberculosis Meningitis and the Use of Unsupervised Isoniazid Prophylaxis. South African Medical Journal, 85, 167-170.

[38] Anochie, I.C. and Nkanginieme, K.E.O. (2000) Prevalence of BCG Skin Reactions and Associated Factors among Children in Port Harcourt Metropolis. Nigerian Journal of Medicine, 4, 134-137.

[39] Middelkoop, K., Bekker, L., Myer, l., Dawson, R. and Wood, R. (2008) Rates of Tuberculosis Transmission to Children and Adolescents in a Community with a High Prevalence of HIV Infection among Adults. Clinical Infectious Diseases, 47, 349-355. http://dx.doi.org/10.1086/589750

[40] Claessens, N.J.M., Gausi, F.F., Meijnen, S., Weismuller, M.M., Salaniponi, F.M. and Harries, A.D. (2002) Screening Childhood Contacts of Patients with Smear-Positive Pulmonary Tuberculosis in Malawi. International Journal of Tuberculosis and Lung Disease, 6, 362-364.

[41] FMOH-NTBLCP/WHO/USAID/TB CARE 1 (2013) Desk Guide for the Diagnosis and Management of TB in Children in Nigeria. FNWHOUTBC 1.

[42] Federal Ministry of Health-National Tuberculosis and Leprosy Control Programme (2015) National Tuberculosis, Leprosy and Buruli Ulcer Management and Control Guidelines, Sixth Edition.

[43] Rook, G.A.W. (1991) Mobilizing the Appropriate T-Cell Subset: The Immune Response as a Taxonomist? Tubercle, 72, 253-254. http://dx.doi.org/10.1016/0041-3879(91)90049-X

[44] Rook, G.A.W. and Al Attiyah, R. (1991) Cytokines and the Koch’s Phenonmenon. Tubercle, 72, 13-20. http://dx.doi.org/10.1016/0041-3879(91)90019-O

[45] Standford, J.L. (1991) Koch’s Phenomenon: Can It Be Corrected? Tubercle, 72, 241-249. http://dx.doi.org/10.1016/0041-3879(91)90047-V

[46] Vallejo, J.G., Ong, L.T. and Starke, J.R. (1994) Clinical Features, Diagnosis and Treatment of Tuberculosis in Infants. Pediatrics, 94, 1-7.

[47] Obafunwa, J.O., Olomu, I.N. and Onyia, N.J. (1992) Primary Peripheral Lymphadenopathy in Jos, Nigeria. West African Journal of Medicine, 11, 25-28.

[48] Aderele, W.I. (1980) Superficial Tuberculosis Lymphadenitis in Childhood. Nigerian Medical Journal, 10, $23-31$.

[49] Anderson, T.S., Kaforou, M., Brent, A.J., Wright, V.J., Banwell, C.M., Chagaluka, G., Crampin, A.C., Dockrell, H.M., French, N., Hamilton, M.S., Hibberd, M.L., Kern, F., Langford, P.R., Ling, L., Mlotha, R., Tom, H.M., Ottenhoff, T.H.M., Pienaar, S., Pillay, V., Scott, J.A.G., Twahir, H., Wilkinson, R.J., Coin, L.J., Heyderman, R.S., Levin, M. and Eley, B. (2014) Diagnosis of Childhood Tuberculosis and Host RNA Expression in Africa. New England Journal of Medicine, 370, 1712-1723. http://dx.doi.org/10.1056/NEJMoa1303657

[50] Ray, S., Talukdar, A., Kimdu, S., Kharma, D. and Sonthalia, S. (2013) Diagnosis and Management of Miliary Tuberculosis: Current State and Future Perspectives. Therapeutics and Clinical Risk Management, 9, 9-26. http://dx.doi.org/10.2147/TCRM.S29179 